\section{Human Motion Tracking by Registering an Articulated Surface to 3-D Points and Normals}

\author{
Radu Horaud, Matti Niskanen, Guillaume Dewaele, and \\ Edmond Boyer
}

\begin{abstract}
We address the problem of human motion tracking by registering a surface to 3-D data. We propose a method that iteratively computes two things: Maximum likelihood estimates for both the kinematic and free-motion parameters of a kinematic human-body representation, as well as probabilities that the data are assigned either to a body part, or to an outlier cluster. We introduce a new metric between observed points and normals on one side, and a parameterized surface on the other side, the latter being defined as a blending over a set of ellipsoids. We claim that this metric is well suited when one deals with either visual-hull or visual-shape observations. We illustrate the method by tracking human motions using sparse visual-shape data (3-D surface points and normals) gathered from imperfect silhouettes.
\end{abstract}

Index Terms-model-based tracking, human motion capture, articulated implicit surface, shape from silhouettes, robust surface registration, expectation-maximization.

\section{INTRODUCTION}

We address the problem of recovering articulated humanmotion parameters using 3-D data gathered from multiple image sequences. We advocate that this type of data has several advantages over 2-D data: it is less ambiguous and it is less sensitive to self-occlusions. 3-D features may be obtained by stereo [12], [5], [6]. Alternatively one can capitalize on 3-D shape from silhouettes. In general, 2-D silhouettes are explicitly associated with a 3-D smooth surface [12], [9], [10]. Another way to use silhouettes is to infer volumetric representations and to fit articulated models to the voxels thus obtained [4], [13], or to extract skeletal representations from these voxels [3]. It is also possible to infer 3-D surfaces from silhouettes, namely the visual hull [2] or the visual shape [8]. The advantage of surface-from-silhouettes is that it allows the recovery of both 3-D surface points and surface normals. Moreover, there is no matching process associated with the reconstruction algorithm. Visual hull algorithms have been proved to be extremely useful for recovering 3-D meshes which, in turn, are very useful for surface rendering. The drawback is that they need perfect silhouettes. Alternatively, visual shape methods (such as the one described in [8]) produce sparse surface descriptions (points and normals) and can operate on imperfect silhouettes.

In this short paper we present a new method for tracking human motion based on fitting an articulated implicit surface to 3-D points and normals. There are two important contributions.

R. Horaud, G. Dewaele, and E. Boyer are with INRIA Grenoble RhôneAlpes, 655 avenue de l'Europe, 38330 Montbonnot Saint-Martin, France.

M. Niskanen is with the Machine Vision Group, Infotech Oulu, University of Oulu, PO Box 4500, Finland. M. Niskanen acknowledges financial support from the Seppo Synjkangas foundation.
First, we introduce a new distance between an observation (a point and a normal) and an ellipsoid. We show that this can be used to define an implicit surface as a blending over a set of ellipsoids which are linked together to from a kinematic chain. Second, we exploit the analogy between the distance from a set of observations to the implicit surface and the negative log-likelihood of a mixture of Gaussian distributions. This allows us to cast the problem of implicit surface fitting into the problem of maximum likelihood (ML) estimation with hidden variables. We argue that outliers are best described by a uniform component that is added to the mixture, and we formally derive the associated EM algorithm.

Casting the data-to-model association problem into ML with hidden variables has already been addressed in the past within the framework of point registration [14], [11], [6]. In [5] observations are deterministically and iteratively assigned to each individual body part. We appear to be the first to apply a probabilistic data-to-model association framework to the problem of fitting a blending of ellipsoids to a set of 3D observations and to explicitly model outliers within this context.

The remainder of the paper is organized as follows. Section II describes how to compute a distance between a 3-D observation (point and normal) and an ellipsoid, and how to build an implicit articulated surface based on this distance. Finally, it introduces the concept of a probabilistic implicit surface. Section III describes the formal derivation of the EM algorithm in the case of implicit surface fitting. Section IV describes experiments with simulated data and with multiplecamera video data.

\section{Modeling ARTiCUlated oBJeCtS}

In order to model articulated objects such as human bodies we must define a number of open kinematic chains that link the various body parts. We will use ellipsoids for modeling these parts. Since we measure 3-D data (point and orientation vectors) we must properly define a metric that measures the discrepancy between the data and the model. This metric will be used to define a distance function as well as a probabilistic implicit surface.

\section{A. The distance from a 3-D datum to an ellipsoid}

One convenient way to describe 3-D ellipsoids is to use an implicit equation and to embed the 3-D Euclidean space into the 3 -D projective space. This yields a $4 \times 4$ full-rank symmetric matrix $\mathbf{Q}$ :

$$
\mathbf{Q}=\left[\begin{array}{cc}
\overline{\mathbf{Q}} & \boldsymbol{q} \\
\boldsymbol{q}^{\top} & q_{44}
\end{array}\right]=\left[\begin{array}{cc}
\mathbf{R D R}^{\top} & -\mathbf{R D R}^{\top} \boldsymbol{t} \\
-\boldsymbol{t}^{\top} \mathbf{R D R}^{\top} & \boldsymbol{t}^{\top} \mathbf{R D R}^{\top} \boldsymbol{t}-1
\end{array}\right]
$$

where $\mathbf{D}=\operatorname{Diag}\left[a^{-2}, b^{-2}, c^{-2}\right]$ is a $3 \times 3$ diagonal matrix, $\mathbf{R}$ is a $3 \times 3$ rotation matrix and $\boldsymbol{t}$ is a $3-\mathrm{D}$ translation vector. In practice $b=c$ and we choose $a \geq b$. We denote by $\boldsymbol{X}$ the homogeneous coordinates of a point $\boldsymbol{x}$ lying onto the surface of the ellipsoid, $\boldsymbol{X}^{\top} \mathbf{Q} \boldsymbol{X}=0$. The adjoint matrix $\mathbf{Q}^{\star}=\mathbf{Q}^{-\top}$ 
defines the dual ellipsoid. The family $\boldsymbol{P}$ of planes which are tangent to the ellipsoid $\mathbf{Q}$ satisfy the constraint $\boldsymbol{P}^{\top} \mathbf{Q}^{-1} \boldsymbol{P}=$ 0 since $\mathbf{Q}^{\top}=\mathbf{Q}$. We denote by $\boldsymbol{p}$ the 3-D vector which is orthogonal to the plane $\boldsymbol{P}$ (therefore $\boldsymbol{p}$ is normal to the ellipsoid at point $\boldsymbol{x}$ ): $\boldsymbol{p}=\overline{\mathbf{Q}} \boldsymbol{x}+\boldsymbol{q}$, where the notations of eq. (1) are used.

The algebraic distance from a 3-D point $\boldsymbol{Y}$ to the surface of an ellipsoid was used in [12], defined by $q(\boldsymbol{Y})=\boldsymbol{Y}^{\top} \mathbf{Q Y}$. The value of $q$ varies from -1 at the center of the ellipsoid, to 0 on its surface, and then to $+\infty$ outside the ellipsoid as the point is farther away from the surface. The Euclidean distance from a point to an ellipsoid requires to solve a six-degree polynomial. In [6] an approximation of the Euclidean distance is used, i.e, a pseudo-Euclidean distance, as shown on Figure 1

An observation will be refered to as a 3-D datum and consists of both a 3-D point and a 3-D vector. We define a metric between such a 3-D datum and an ellipsoid as follows. Let $\boldsymbol{Y}^{\top}=\left(\boldsymbol{y}^{\top} 1\right)$ be the homogeneous coordinates of an observed point, and let $\boldsymbol{n}$ be a 3-D observed vector. An observation or a 3-D datum is denoted by $\mathcal{Y}=(\boldsymbol{y}, \boldsymbol{n})$. We seek an ellipsoid point $\boldsymbol{X}=\left(\boldsymbol{x}^{\top} 1\right)$ under the constraint that the vector $\boldsymbol{p}$ (normal to the ellipsoid at $\boldsymbol{x}$ ) is aligned with $\boldsymbol{n}$, e.g. Figure 1 In other words, we seek an association between $\mathcal{X}=(\boldsymbol{x}, \boldsymbol{p})$ and $\mathcal{Y}=(\boldsymbol{y}, \boldsymbol{n})$. Figure 2 compares the distance used in this paper with the Euclidean distance from a point to an ellipsoid.

Let $d_{\mathcal{E}}(\mathcal{Y}, \mathcal{X})$ be the Euclidean distance from the datumpoint $\boldsymbol{y}$ to the ellipsoid-point $\boldsymbol{x}$ under the constraint that the datum-vector $\boldsymbol{n}$ and the ellipsoid-vector $\boldsymbol{p}$ are parallel:

$$
d_{\mathcal{E}}(\mathcal{Y}, \mathcal{X})=\|\boldsymbol{x}-\boldsymbol{y}\|_{2} \text { with } \boldsymbol{n} \times \boldsymbol{p}=0
$$

where $\|\boldsymbol{a}\|_{2}$ denotes the Euclidean norm. We seek a solution for $\boldsymbol{x}$ under the constraints that $\boldsymbol{p}$ and $\boldsymbol{n}$ are parallel and yield the same orientation. Using eq. (1) we obtain the following set of constraints:

$$
\begin{aligned}
\boldsymbol{x}^{\top} \overline{\mathbf{Q}} \boldsymbol{x}+2 \boldsymbol{x}^{\top} \boldsymbol{q}+q_{44} & =0 \\
\overline{\mathbf{Q}} \boldsymbol{x}+\boldsymbol{q} & =\lambda \boldsymbol{n}
\end{aligned}
$$

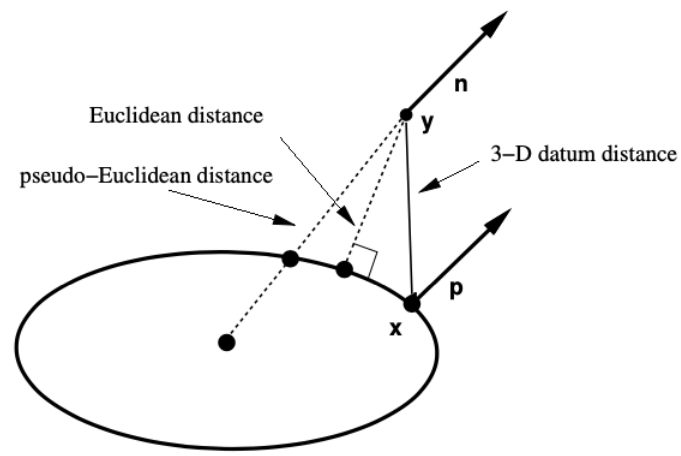

Fig. 1. The distance from the datum $\mathcal{Y}=(\boldsymbol{y}, \boldsymbol{n})$ to the ellipsoid $\mathbf{Q}$ is estimated by seeking the point $\boldsymbol{x} \in \mathbf{Q}$ such that the normal $\boldsymbol{p}$ at $\boldsymbol{x}$ is aligned with vector $\boldsymbol{n}$.
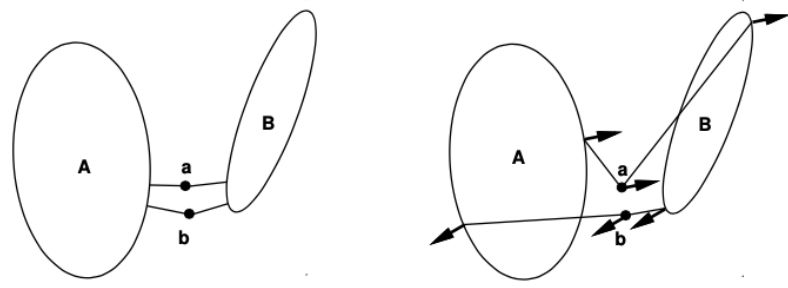

Fig. 2. The classical Euclidean distance from a point to an ellipsoid (left) does not assign a point to an ellipsoid in an unambiguous way. The 3-D datum distance (right) assigns without ambiguity $\boldsymbol{a}$ to $\mathbf{A}$ and $\boldsymbol{b}$ to $\mathbf{B}$.

From eq. 44) we obtain $\boldsymbol{x}=\overline{\mathbf{Q}}^{-1}(\lambda \boldsymbol{n}-\boldsymbol{q})$. By substitution in eq. (3) we obtain two solutions for $\lambda$. From $\boldsymbol{p}^{\top} \boldsymbol{n}>0$ we have $\lambda>0$ and $\lambda=\left(\boldsymbol{n}^{\top} \mathbf{R D}^{-1} \mathbf{R}^{\top} \boldsymbol{n}\right)^{-1 / 2}$. Therefore, the point onto the ellipsoid where its normal $\boldsymbol{p}$ is aligned with $\boldsymbol{n}$ is given by:

$$
\boldsymbol{x}=\lambda \mathbf{R} \mathbf{D}^{-1} \mathbf{R}^{\top} \boldsymbol{n}+\boldsymbol{t}
$$

It will be convenient to use the Mahalanobis distance:

$$
d_{\mathcal{M}}^{2}(\boldsymbol{y}, \boldsymbol{x})=(\boldsymbol{y}-\boldsymbol{x}(\mathbf{R}, \boldsymbol{t}, \boldsymbol{n}))^{\top} \Sigma^{-1}(\boldsymbol{y}-\boldsymbol{x}(\mathbf{R}, \boldsymbol{t}, \boldsymbol{n}))
$$

\section{B. Kinematic chains and human-body modeling}

Articulated motion has a long history in mechanics, biomechanics, robotics, and computer vision. A human body can be described by a number of open kinematic chains that share a common root. Such an open chain is composed of a number of rigid objects and two consecutive rigid objects in the chain are mechanically linked to form a joint. Rotational (or spherical) joints are the most convenient representations and they are well suited for human body modeling. Each such joint may have one, two, or three rotational degrees of freedom. Therefore, within such a chain, a body part $\mathbf{Q}$ is linked to a root body part $\mathbf{Q}_{r}$ through a constrained motion, i.e., a kinematic chain with a number of rotational degrees of freedom. Since each joint may have several degrees of freedom, the total number of rotational parameters of a chain is larger than the number of rigid parts composing the chain. Moreover, the root body part undergoes a free motion itself, i.e., a rigid displacement with up to six degrees of freedom: three rotations and three translations.

Therefore the motion of a body-part (or ellipsoid) $\mathbf{Q}$ is composed of the root's free motion followed by the chain's constrained motion. We will denote the motion of $\mathbf{Q}$ by the $4 \times 4$ homogeneous matrix $\mathbf{T}$ which in turn is parameterized by the joint and free-motion parameter vector $\Lambda$ :

$$
\mathbf{T}(\boldsymbol{\Lambda})=\left[\begin{array}{cc}
\mathbf{R}(\boldsymbol{\Lambda}) & \boldsymbol{t}(\boldsymbol{\Lambda}) \\
\mathbf{0} & 1
\end{array}\right]
$$

A complete human-body model may be described with five kinematic chains that share a common root body-part. In this paper we use the following simplified human-body model. There are 14 body parts and 11 joints ( 2 ankles, 2 knees, 
2 hips, 2 elbows, 2 shoulders, and a neck) with 22 rotational degrees of freedom (there are two degrees of freedom for each joint). We also consider 3 rotations and 3 translations for the free motion. Hence, there is a total of 26 degrees of freedom.

As detailed above, body parts are described by one or several ellipsoids: The feet and the thighs are described by two ellipsoids, the torso is described by three ellipsoids, and all the other body parts are described by a single ellipsoid, hence there are 21 ellipsoids, e.g., Figure 3 . The body parts are denoted by $\mathbf{Q}_{p}, 1 \leq p \leq P$, where, for convenientce, $\mathbf{Q}_{1}$ corresponds to the common root body part.
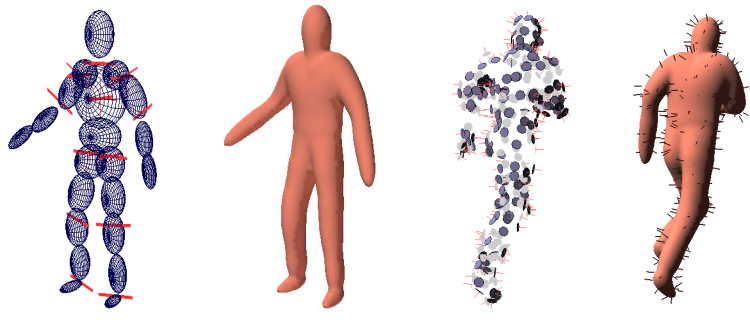

Fig. 3. From left to right: The set of 21 ellipsoids used to model 14 body parts with 11 joints and 2 rotations per joint. The implicit surface defined as a blending of these ellipsoids. A set of 3-D "surface" observations (points and normals) and the articulated implicit surface that has been fitted to these observations.

\section{Articulated implicit surfaces}

In addition to using a collection of kinematically linked ellipsoids, we will fuse them in order to define a smooth surface $\mathcal{S}$ over the entire body. This surface will be described by the implicit equation $f(\boldsymbol{y})=C$ with $C=1$. The contribution of an ellipsoid $\mathbf{Q}_{p}$ is defined by:

$$
f_{p}(\boldsymbol{y})=\exp \left(-\frac{d_{\mathcal{M}}^{2}\left(\boldsymbol{y}, \boldsymbol{x}_{p}\right)}{\nu_{p}^{2}}\right)
$$

where $d_{\mathcal{M}}$ is the Mahalanobis distance from $\boldsymbol{y}$ to the ellipsoid defined by eq. 6, the point $\boldsymbol{x}_{p}$ lies onto the ellipsoid, and $\nu_{p}^{2}$ is a parameter that tunes the spatial influence of the ellipsoid.

An implicit surface is defined as a level set of the following implicit function which is the fusion (or blending) of $P$ ellipsoids verifying:

$$
f(\boldsymbol{y})=\sum_{p=1}^{P} f_{p}(\boldsymbol{y})
$$

The class of implicit surfaces defined as above, i.e., $\boldsymbol{y} \in \mathcal{S} \Leftrightarrow$ $f(\boldsymbol{y})=C$, has successfully been used in computer graphics and in computer vision in conjunction with the algebraic distance [12] and with the pseudo-Euclidean distance [6]. Within this paper we extend this concept to the distance defined above. As it will be detailed below, this is well suited to cast the problem of implicit surface fitting into the framework of maximum likelihood in the presence of outliers.

In order to track articulated objects, the task at hand consists of fitting the articulated implicit surface just described to a set of observations. For this purpose, we first define a distance from a set of observations to the implicit surface. We have to solve the equation $f(\boldsymbol{y})=1$, where $\boldsymbol{y}$ is, as before, an observed 3-D point. One may notice that the first order Taylor expansion of $\ln a$ at $a=1$ is: $\ln a=a-1+O\left(a^{2}\right)$. We retain the following approximation of the distance from a set of $I$ observations to the articulated implicit surface formed by $P$ ellipsoids and parameterized by the kinematic variables $\Lambda$ :

$$
F(\boldsymbol{\Lambda})=-\nu^{2} \sum_{i=1}^{I} \ln \sum_{p=1}^{P} \exp \left(-\frac{d_{i p}^{2}}{\nu^{2}}\right)
$$

where:

$$
d_{i p}^{2}=\left(\boldsymbol{y}_{i}-\boldsymbol{x}_{i p}\left(\boldsymbol{\Lambda}, \boldsymbol{n}_{i}\right)\right)^{\top} \Sigma_{p}^{-1}\left(\boldsymbol{y}_{i}-\boldsymbol{x}_{i p}\left(\boldsymbol{\Lambda}, \boldsymbol{n}_{i}\right)\right)
$$

For convenience, we set $\nu=\nu_{1}=\ldots=\nu_{P}$. The notation $\boldsymbol{x}_{i p}$ means that the 3-D point $\boldsymbol{x}$ lies on ellipsoid $p$ and is associated with observation $i$. It is worthwhile to notice that, whenever a set of observations is closed to one of the ellipsoids, the distance function is strictly equal to the sum of Mahalanobis distances from each such observation to the ellipsoid.

\section{Probabilistic implicit surfaces}

In this section we introduce a probabilistic interpretation of eq. (10). For this purpose we denote by $z_{i}$ a random variable that assigns an observation $i$ to an ellipsoid $p$, namely the notation $z_{i}=p$ means that the $i^{\text {th }}$ observation is assigned to the $p^{\text {th }}$ ellipsoid. There are as many hidden variables $z_{i}$ as observations: $i \in\{1, \ldots, I\}$. The set of all the hidden variables is denoted by $\mathcal{Z}=\left\{z_{1}, \ldots, z_{I}\right\}$.

The likelihood of an observed 3-D point, given its assignment to an ellipsoid and given an observed 3-D normal, is drawn from a Gaussian distribution:

$$
P\left(\boldsymbol{y}_{i} \mid z_{i}=p, \boldsymbol{n}_{i}\right)=\mathcal{N}\left(\boldsymbol{y}_{i} \mid \boldsymbol{x}_{i p}\left(\boldsymbol{\Lambda}, \boldsymbol{n}_{i}\right), \Sigma_{p}\right)
$$

In practice the data are corrupted by noise and by errors and therefore there are observations which should not be assigned to an ellipsoid. For this reason we introduce an outlier class denoted by $P+1$, and we assume that the likelihood of an observation given that is classified as an outlier is a uniform distribution over the volume $V$ of the working space:

$$
P\left(\boldsymbol{y}_{i} \mid z_{i}=P+1, \boldsymbol{n}_{i}\right)=\mathcal{U}\left(\boldsymbol{y}_{i} \mid V, 0\right)=\frac{1}{V}
$$

Therefore, one can write the likelihood of an observation as a mixture of $P$ Gaussian components and one uniform component:

$$
P\left(\boldsymbol{y}_{i} \mid \boldsymbol{n}_{i}\right)=\sum_{p=1}^{P+1} \pi_{p} P\left(\boldsymbol{y}_{i} \mid z_{i}=p, \boldsymbol{n}_{i}\right)
$$

The notation:

$$
\pi_{p}=P\left(z_{i}=p \mid \boldsymbol{n}_{i}\right)
$$

denotes the priors, the proportions, or the mixing parameters, and they obey the obvious constraint $\sum_{p=1}^{P+1} \pi_{p}=1$. Notice that this prior probability depends on the observed vector 
$\boldsymbol{n}_{i}$. In this paper we do not treat these observed vectors as random variables. By assuming independent and identically distributed observations one can write the joint likelihood of all the observations as:

$P\left(\mathcal{Y}_{1}, \ldots, \mathcal{Y}_{I}\right)=P\left(\boldsymbol{y}_{1}, \boldsymbol{n}_{1}, \ldots, \boldsymbol{y}_{I}, \boldsymbol{n}_{I}\right)=\prod_{i=1}^{I} P\left(\boldsymbol{y}_{i} \mid \boldsymbol{n}_{i}\right) P\left(\boldsymbol{n}_{i}\right)$

Using Bayes' formula and the equations above, the negative log-likelihood writes:

$$
\begin{aligned}
& -\ln P_{\boldsymbol{\Lambda}}\left(\mathcal{Y}_{1}, \ldots, \mathcal{Y}_{I}\right)= \\
& -\sum_{i=1}^{I} \ln \left(\sum_{p=1}^{P} \pi_{p} \mathcal{N}\left(\boldsymbol{y}_{i} \mid \boldsymbol{x}_{i p}\left(\boldsymbol{\Lambda}, \boldsymbol{n}_{i}\right), \Sigma_{p}\right)\right. \\
& +\quad \pi_{P+1} \mathcal{U}\left(\boldsymbol{y}_{i} \mid V, 0\right)
\end{aligned}
$$

Notice that there is a strong analogy between eqs. (10) and (15): the former is a distance between a set of $I$ observations and an articulated implicit surface while the latter is the joint likelihood of the same observation set, where the likelihood is a mixture of $P$ normal distributions plus a uniform distribution that captures the bad observations. This analogy will be exploited in the next section in order to cast the estimation of the kinematic parameters in the framework of maximum likelihood with hidden variables via the EM algorithm.

\section{ROBUST TRACKING WITH THE EM ALGORITHM}

Because of the presence of the hidden variables, $\mathcal{Z}=$ $\left\{z_{1}, \ldots, z_{I}\right\}$, the maximum-likelihood estimation problem, i.e., eq. (15) does not have a simple solution. The most convenient way to maximize the likelihood of a mixture of distributions is to use the EM algorithm. The latter has been thoroughly studied in the context of data clustering [7]. In this paper we formally derived an expectation-maximization scheme in the particular case of robustly fitting an implicit surface to a set of 3-D observations. It is worthwhile to notice that the formulae below are valid independently of the distance function being used, i.e., Figure 1 .

First we derive the posterior class probabilities conditioned by the observations, namely:

$$
P\left(z_{i}=p \mid \boldsymbol{y}_{i}, \boldsymbol{n}_{i}\right)=\frac{P\left(z_{i}=p, \boldsymbol{y}_{i}, \boldsymbol{n}_{i}\right)}{P\left(\boldsymbol{y}_{i}, \boldsymbol{n}_{i}\right)}
$$

We denote these posteriors by $t_{i p}$ and with the notations introduced in the prevous section we obviously obtain:

$$
t_{i p}=\frac{\pi_{p} P\left(\boldsymbol{y}_{i} \mid z_{i}=p, \boldsymbol{n}_{i}\right)}{P\left(\boldsymbol{y}_{i} \mid \boldsymbol{n}_{i}\right)}
$$

Second we consider the joint probability of the set of observations $\mathcal{Y}=\left\{\mathcal{Y}_{1}, \ldots, \mathcal{Y}_{I}\right\}$ and of their assignments $\mathcal{Z}$ which yield the following expression:

$P(\mathcal{Y}, \mathcal{Z})=\prod_{i=1}^{I} \prod_{p=1}^{P+1}\left(P\left(\boldsymbol{y}_{i} \mid z_{i}=p, \boldsymbol{n}_{i}\right) P\left(z_{i}=p \mid \boldsymbol{n}_{i}\right)\right)^{\delta_{p}\left(z_{i}\right)} P\left(\boldsymbol{n}_{i}\right) \begin{aligned} & \text { Since we formally derived eq. } \\ & \text { below guarantees likelihood the EM algorithm outlined }\end{aligned}$ with the following definition for the function $\delta_{p}\left(z_{i}\right)$ :

$$
\delta_{p}\left(z_{i}\right)=\left\{\begin{array}{ccc}
1 & \text { if } & z_{i}=p \\
0 & & \text { otherwise }
\end{array}\right.
$$

Third we derive the expression of the conditional expectation of the $\log$-likelihood taken over $\mathcal{Z}$, which in this case yields:

$$
\begin{array}{r}
E[\ln P(\mathcal{Y}, \mathcal{Z}) \mid \mathcal{Y}]= \\
\sum_{i=1}^{I} \sum_{p=1}^{P+1} E\left[\delta_{p}\left(z_{i}\right) \mid \mathcal{Y}\right]\left(\ln P\left(\boldsymbol{y}_{i} \mid z_{i}=p, \boldsymbol{n}_{i}\right)\right. \\
\left.+\ln \pi_{p}\right)+(P+1) \sum_{i=1}^{I} \ln P\left(\boldsymbol{n}_{i}\right)
\end{array}
$$

One may notice that:

$$
E\left[\delta_{p}\left(z_{i}\right) \mid \mathcal{Y}\right]=\sum_{p=1}^{P+1} \delta_{p}\left(z_{i}=p\right) P\left(z_{i}=p \mid \boldsymbol{y}_{i}, \boldsymbol{n}_{i}\right)=t_{i p}
$$

By using the expressions of the normal and uniform distributions, and by grouping constant terms we obtain:

$$
\begin{aligned}
& E[\ln P(\mathcal{Y}, \mathcal{Z}) \mid \mathcal{Y}]= \\
- & \frac{1}{2} \sum_{i=1}^{I}\left(\sum _ { p = 1 } ^ { P } t _ { i p } \left(\left(\boldsymbol{y}_{i}-\boldsymbol{x}_{i p}\right)^{\top} \Sigma_{p}{ }^{-1}\left(\boldsymbol{y}_{i}-\boldsymbol{x}_{i p}\right)\right.\right. \\
+ & \left.\left.\ln \operatorname{det} \Sigma_{p}-\ln \pi_{p}\right)+t_{i P+1} \ln \pi_{P+1}\right)+\operatorname{const}(17)
\end{aligned}
$$

The maximization of eq. (17) (or equivalently the minimization of its negative) will be carried out via the EM algortihm (expectation-maximization). There are, however three notable differences between the standard EM for Gaussian mixtures [1] and our formulation:

- We added a uniform-noise component to the mixture. The role of this component is to "capture" outliers and hence to avoid that they influence the estimation of the model parameters;

- The means of the Gaussian components, $\boldsymbol{x}_{i p}$ are parameterized by the kinematic parameters that control the articulated motion of each ellipsoid; This has an important consequence because the M-step of the algorithm will incorporate a non-linear minimization procedure over the kinematic joints.

- At the start of the algorithm each observation is associated with all the ellipsoids. As the algorithm proceeds, each observation is eventually associated with one of the ellipsoids. Due to occlusions, missing data, etc., there may be ellipsoids with no associated observation. Therefore, there is a risk that the corresponding covariance becomes infinitely small. To overcome this problem we use a unique covariance matrix common to all the densities in the mixture. 
the advantages of this formulation are (i) fast convergence properties of EM and (ii) the fact that it minimizes the negative log-likelihood given by eqs. (15) and (17). In practice, the following EM procedure can be used for robust tracking of an articulated implicit surface:

1) Initialization. Compute the locations of the ellipsoid points $\boldsymbol{x}_{i p}^{(q)}$ from the current kinematic parameters $\boldsymbol{\Lambda}^{(q)}$ using eq. (5). Similarly, initialize the covariance matrix $\Sigma^{(q)}$ common to all the ellipsoids. Initialize the priors, or the mixing parameters $\pi_{1}^{(q)}=\ldots=\pi_{P+1}^{(q)}=1 /(P+1)$;

2) E step. Evaluate the posterior probabilities $t_{i p}^{(q)}$ using the current parameter values, through eq. (16);

3) M step. Estimate new values for the kinematic parameters $\boldsymbol{\Lambda}^{(q+1)}$

$\arg \min _{\boldsymbol{\Lambda}} \frac{1}{2} \sum_{i=1}^{I} \sum_{p=1}^{P} t_{i p}^{(q)}\left(\boldsymbol{y}_{i}-\boldsymbol{x}_{i p}(\boldsymbol{\Lambda})\right)^{\top} \Sigma^{(q)^{-1}}\left(\boldsymbol{y}_{i}-\boldsymbol{x}_{i p}(\boldsymbol{\Lambda})\right)$

Update the covariance matrix and the priors:

$$
\begin{aligned}
\Sigma^{(q+1)} & = \\
& \frac{1}{\sum_{p=1}^{P} T_{p}} \sum_{i=1}^{I} \sum_{p=1}^{P} t_{i p}^{(q)}\left(\boldsymbol{y}_{i}-\boldsymbol{x}_{i p}\left(\boldsymbol{\Lambda}^{(q+1)}\right)\right) \\
& \left(\boldsymbol{y}_{i}-\boldsymbol{x}_{i p}\left(\boldsymbol{\Lambda}^{(q+1)}\right)\right)^{\top} \\
\pi_{p}^{(q+1)} & =\frac{1}{I} \sum_{i=1}^{I} t_{i p}^{(q)}
\end{aligned}
$$

4) Maximum likelihood. Evaluate the log-likelihood, i.e., eq. 15) and check for convergence.

\section{EXPERIMENTAL RESULTS}

The tracking algorithm described in the previous section is not tight to any particular method for extracting the 3-D data. In practice we used 3-D points and normals that lie on the visual shape [8]. Notice that the visual-shape algorithm does not require perfect silhouettes and provides as output a sparse set of 3-D points and normals, not a 3-D mesh. The visual-shape method uses multiple-view epipolar geometry in conjunction with the assumption that the object's surface is locally continuous and twice differentiable (see [8] for details).

\section{A. Experiments with simulated data}

We used an animation package to build a human body, to simulate various human motions, and to render image silhouettes. The simulator uses its own shape representation, that is different than ours, but it allows the user to define her/his own kinematic model. Therefore we used the same kinematic model with the same number of degrees of freedom as the one described in section II-B Nevertheless, we have not attempted to finely tune the shape parameters of our model to the simulated data. We simulated a setup composed of seven calibrated cameras. Sequences of image silhouettes were generated from the 3-D model and rendered with the cameras' parameters. We computed 3-D points and normals from these silhouettes and then we applied our method to these data sets. The articulated-motion parameters were recovered using our tracker. In order to assess the merits of the data-to-model fitting process, we added $20 \%$ of outliers uniformly distributed in the volume of the working space. These simulations allowed us to (i) assess the quality of the tracker with respect to groundtruth joint trajectories, (ii) analyse the behavior of the method in the presence of various perturbations that alter the quality of the data, (iii) quantify the merit of using both 3-D points and normals instead of using only points, and (iv) determine the optimal number of observations needed for reliable estimation of articulated motion.
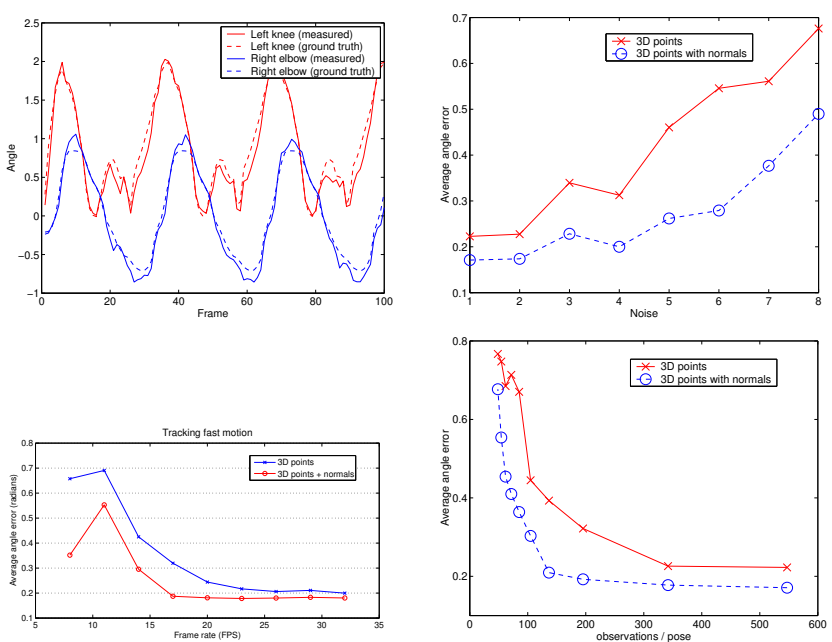

Fig. 4. The error between simulated angle values and estimated ones (measured in radians), from left ot right: Ground-truth and measured trajectories over 100 frames. Average angle error as a function of silhouette noise for points and for points and normals. Average angle error as a function of frame rate. Average angle error as a function of the number of observations being used.

We simulated a running sequence that involves both kinematic and free-motion parameters. The graphs of Figure 4 illustrate the average error between the simulated angle values and their estimated values (measured in radians) for the kinematic parameters but not for the free-motion parameters. The first graph compares the ground-truth (simulated) joint trajectories of the left knee and of the right elbow (dashed curve) with the trajectories estimated with our method (solid curve) over 100 frames. The second graph illustrates the behavior of the method in the presence of silhouette noise. The results of using both 3-D points and normals (dashed curve) are plotted against the results obtained using 3-D points and the algebraic distance (solid line). The relative large error corresponds to the fact that the shape model used by the animation package is not the same as our shape model. Hence, there is a systematic offset between the ground-truth kinematic parameters and the estimated parameters.

The third graph shows the average angle error as a function of the number of frames per second. The last graph shows the influence of the number of observations, where the latter varies from 50 to 550 . The average angle error drops as the number of observations increases and our method (dashed) performs better than using 3-D points alone (solid). From all 

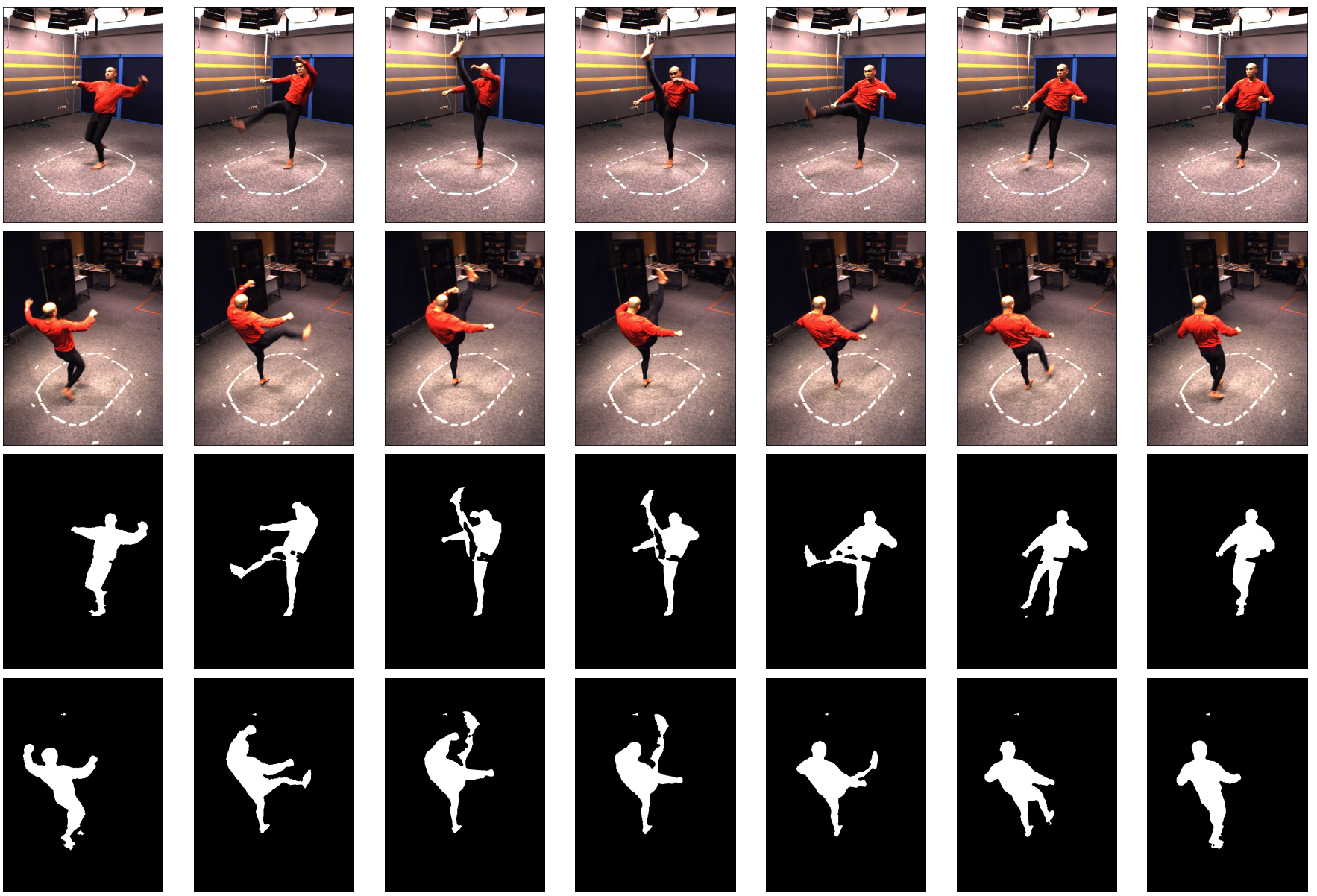

Fig. 5. The taekwendo sequence. The images and the associated silhouettes are from the first and fourth cameras. Imperfect silhouettes generate outlier data which are properly handled by our method.

these experiments one may conclude that tracking is improved when both points and normals are used instead of just points.

\section{B. Experiments with multiple-video data}

The experimental data that we used for validating the human motion tracker was gathered with six calibrated and finely synchronized cameras. Each camera delivers $780 \times 580$ color images at 28 frames per second with a synchronization accuracy within 1 mili-second. The figures below show these image sequences sampled at 14 frames per second.

We applied articulated human motion tracking to two multiple-image sequences, the taekwendo sequence shown on Figure 5 and the leaning sequence shown of Figure 7. The first data set is composed of $6 \times 700$ frames while the second one is composed of $6 \times 200$ frames. We used the same bodypart dimensions for the two characters. One may notice that the silhouettes have holes and missing pieces, which results in the presence of 3-D outliers. The top row in Figure 6 shows the 3-D points and normals that were reconstructed from the imperfect silhouettes; The middle row shows the articulated implicit surface resulting from application of our method, while the bottom row shows the same surface resulting from application of our method in conjunction with the algebraic distance. Obviously, in this last case, there is a discrepancy between the data and the fitted model: the recovered motion of the right feet and the right thigh are incorrect. Similarly, figure 7 shows a sample of the leaning sequence, the corresponding silhouettes, and the fitted model using the proposed method.

\section{CONCLUSIONS}

In this paper we described a method for tracking articulated motion with several cameras. We introduced a new metric that measures the discrepancy between observations (composed of both 3-D points and 3-D normals) and an articulated implicit surface. This metric is more powerful than previously used distance functions because it allows for less ambiguous associations between the data and the model. Moreover, it is well suited when one deals with either visual-hull or visualshape representations of the data.

We cast the data-to-model fitting process into a robust probabilistic framework. We showed that there is a strong similarity between the mathematical representation of an implicit surface and a mixture of Gaussian distributions. We explored this similarity and we showed that the articulated motion tracking 


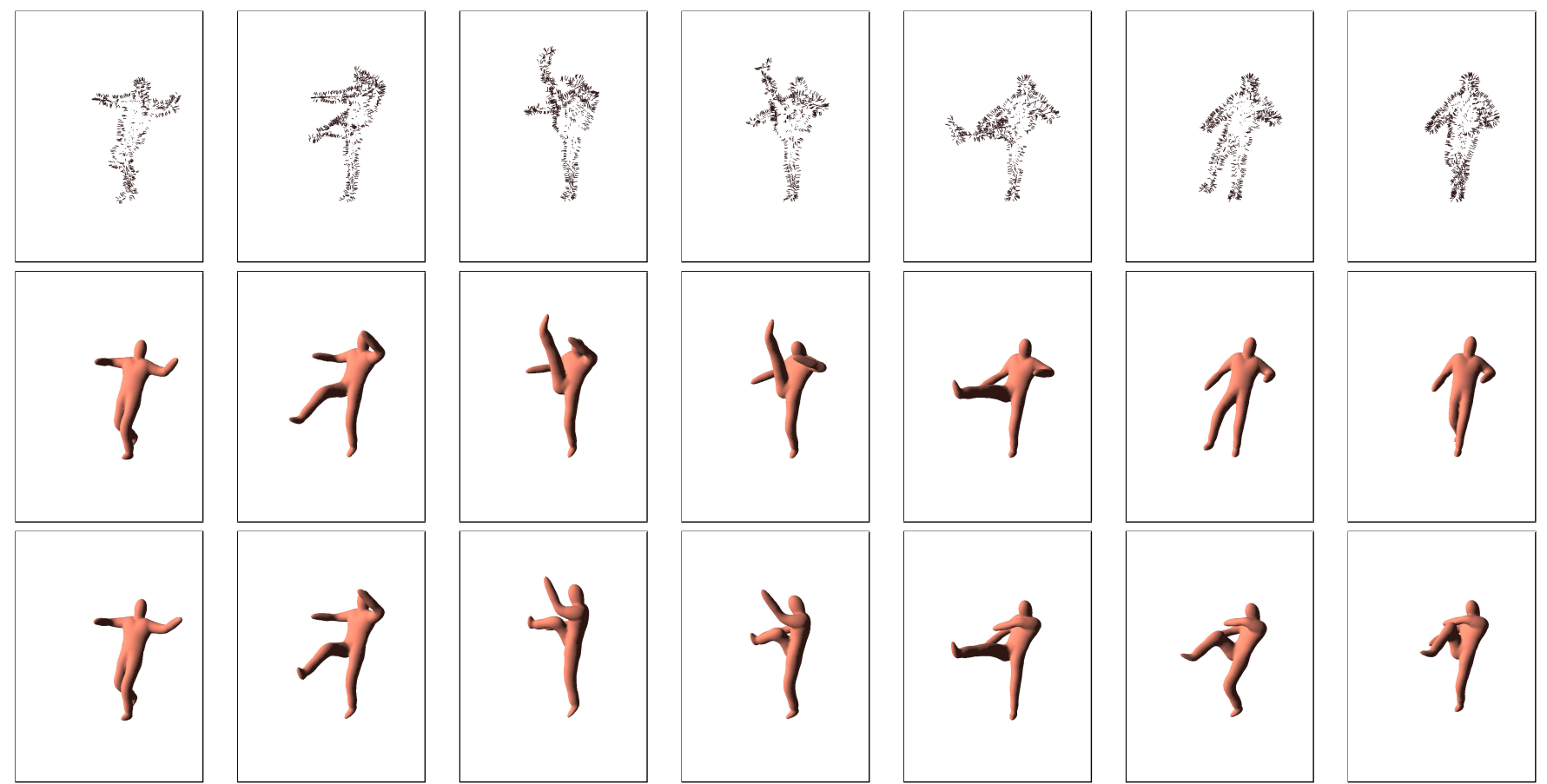

Fig. 6. The 3-D visual hull (top), the implicit surface fitted to the taekwendo sequence using points and normals (middle), and using points alone (bottom).
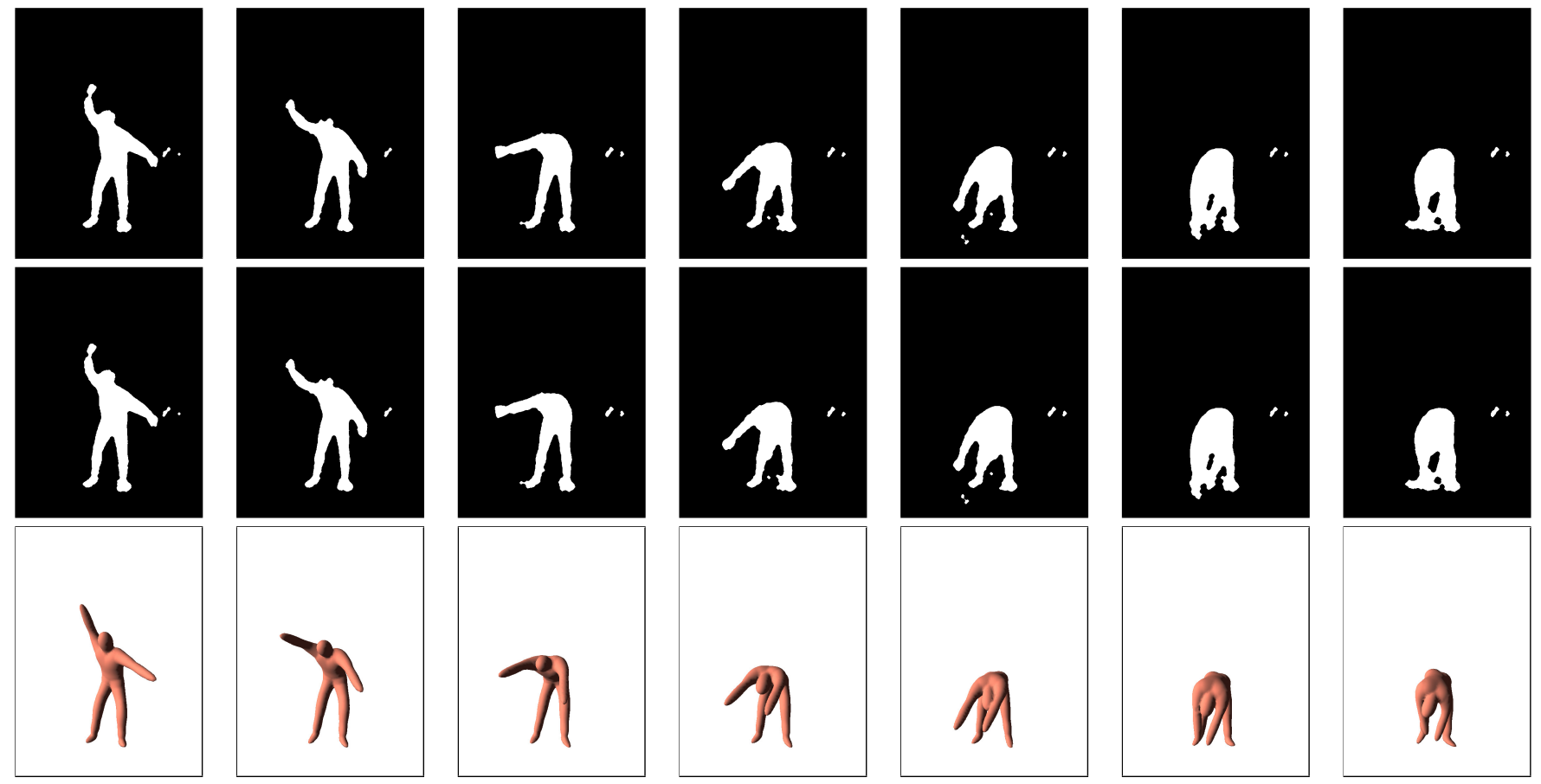

Fig. 7. The leaning sequence: Images and silhouettes associated with the first camera, and the fitted implicit surface.

problem can be formulated as maximum likelihood with hidden variables. We added a uniform component to the mixture to account for outliers. We formally derived an algorithm that computes ML estimates for the motion parameters within the framework of expectation-maximization (EM). Therefore, the tracker may well be interpreted in the framework of robust data clustering, where the observations are assigned to one of the ellipsoids, or to an outlier component.
There are many questions that remain open and that we plan to investigate in the near future: The algorithm may be trapped in local minima if it is not properly initialized; There are some similarities between our robust tracker and the use of Mestimators and these similarities diserve further investigation. There are other interesting issues such as: A thorough and quantitative evaluation of the results and their comparison with marker-based motion capture systems and the possibility to 
capture several articulated motions at once.

\section{REFERENCES}

[1] C.M. Bishop. Pattern Recognition and Machine Learning. Springer, 2006.

[2] E. Boyer and J-S. Franco. A hybrid approach for computing visual hulls of complex objects. In Proceedings of the IEEE Conference on Computer Vision and Pattern Recognition, pages 695-701, Madison, Wisconsin, USA, June 2003. IEEE Computer Society Press.

[3] G.J. Brostow, I.A. Essa, D. Steedly, and V. Kwatra. Novel skeletal representation for articulated creatures. In European Conference on Computer Vision, pages Vol III: 66-78, 2004.

[4] K.-M. Cheung, S. Baker, and T. Kanade. Shape-from-silhouette across time part ii:applications to human modeling and markerless motion. International Journal of Computer Vision, 63(3):225-245, 2005.

[5] D. Demirdjian. Combining geometric- and view-based approaches for articulated pose estimation. In European Conference on Computer Vision, pages Vol III: 183-194, 2004.

[6] G. Dewaele, F. Devernay, R. Horaud, and F. Forbes. The alignment between 3-d data and articulated shapes with bending surfaces. In Proceedings of the 9th European Conference on Computer Vision, Graz, Austria, volume III of LNCS, pages 578-591. Springer, May 2006.

[7] C. Fraley and A. E. Raftery. Model-based clustering, discriminant analysis, and density estimation. Journal of the American Statistical Association, 97:611-631, 2002.

[8] J-S. Franco, M. Lapierre, and E. Boyer. Visual shapes of silhouette sets. In Proceedings of the 3rd International Symposium on $3 D$ Data Processing, Visualization and Transmission, Chapel Hill (USA), 2006.

[9] S. Ilic, M. Salzmann, and P. Fua. Implicit meshes for effective silhouette handling. International Journal of Computer Vision, 72(2):159 - 178, 2007.

[10] D. Knossow, R. Ronfard, and R. Horaud. Human motion tracking with a kinematic parameterization of extremal contours. International Journal of Computer Vision, 2008. To appear.

[11] B. Luo and E.R. Hancock. Structural graph matching using the EM algorithm and singular value decomposition. IEEE Transactions on Pattern Analysis and Machine Intelligence, 23(2001):1120-1136, October 2001.

[12] R. Plankers and P. Fua. Articulated Soft Objects for Multi-View Shape and Motion Capture. IEEE Transactions on Pattern Analysis and Machine Intelligence, 25(9):1182-1187, 2003.

[13] J. Starck and A. Hilton. Model-based multiple view reconstruction of people. In IEEE International Conference on Computer Vision, October 2003.

[14] W.M. Wells III. Statistical approaches to feature-based object recognition. International Journal of Computer Vision, 28(1/2):63-98, 1997. 